\title{
Optimisation of monitoring data for increased predictive reliability of regional water allocation models
}

\author{
$\underline{\text { C.R. Moore }}^{\text {a }}$, Th. Wöhling ${ }^{\text {b,c }}$, L. Wolf ${ }^{\text {a }}$ \\ ${ }^{a}$ Commonwealth Scientific and Industrial Research Organisation (CSIRO), Land and Water Division \\ ${ }^{b}$ Water\&Earth System Science Research Centre (WESS,)University of Tuebingen \\ c Lincoln Ventures (LVL), Private Bag 3062, Hamilton, New Zealand \\ Email: Catherine.moore@csiro.au
}

This paper discusses the optimization of monitoring data for the increased reliability of regional groundwater models and the predictions that depend on them. The significant costs of commissioning and maintaining groundwater monitoring networks are such that there is great benefit in being able to assess where data gathering has the greatest impact on improving predictive reliability. This optimization assessment can be made on the basis of existing networks or prior to any data acquisition efforts. Various data acquisition strategies, for quite disparate data types, can be compared in terms of their ability to increase the reliability of model based predictions; data collection strategies which provide the greatest return for investment can then be selected for implementation. Similarly the relative merits of making measurements at different locations and times can be assessed. Using the Lockyer Valley ground water model (RPS 2010) we demonstrate how predictive uncertainty analysis can provide a powerful foundation for optimizing both existing monitoring networks and future data acquisition strategies to support model based environmental management.

Such analyses are efficient yet robust. The particular characterization of model predictive variance in the problem formulation employed (Moore and Doherty, 2005), ensures that the contributions to predictive uncertainty by both measurement errors and environmental heterogeneity that cannot be captured by the calibration process is accounted for in the analysis. Efficiency is gained via a linearity assumption in the equation used in the analysis, which allows the calculation to be made sufficiently rapidly, so that it can be repeated at many alternative existing or proposed monitoring sites and times. Furthermore, this analysis has no cost barriers, as the software for such analyses is in the public domain (Doherty, 2011a and b). These are particularly important benefits in the large scale regional model context, where monitoring is typically a significant effort and is subject to public scrutiny in terms of both cost and rigour.

Keywords: Optimisation, monitoring networks, groundwater, decision making, predictive uncertainty 


\section{INTRODUCTION}

Regional groundwater models are frequently used as a tool to assist water allocation and/or land-use management. Typically they involve painstaking conceptualization and costly monitoring and data processing efforts before a model can be used to predict the consequences of a proposed suite of management options. Inevitably these predictive simulations will be made with varying degrees of reliability, or uncertainty. In this paper we demonstrate a methodology which can be used to identify optimal data monitoring networks, where "optimal" is defined in terms of increased reliability of the particular prediction that underpins a management decision. Because this analysis does not rely on the actual value of a measurement, but rather the sensitivity of the prediction to the measurement being made, this monitoring data may be an actual measurement or a proposed one. Similarly, data of different types can be easily compared e.g. from pump testing, groundwater water levels, surface water flows, tracer test concentrations etc. Furthermore the analysis can be undertaken both prior to a model meeting calibration constraints (e.g. calibration), or refined after calibration.

The methodology is based on assessing 'data worth' using a predictive error variance analysis theory presented in Moore and Doherty (2005), and a Bayesian extension of this work presented in Christensen and Doherty (2008). This approach for assessing data worth was applied to a synthetic numerical groundwater model in Moore (2005), where the worth of spatially distributed head and hydraulic conductivity measurements were compared, in terms of improvements in reliability of a contaminant transport prediction. The method has since been applied to a number of other synthetic problems, including Dausman et al. (2010) who compared the relative worth of salinity and temperature observations for predictions of the location of a salt water-fresh water interface, Turnadge (2010) who compared the worth of isotope and head measurements for contaminant transport predictions, and Fienen et al. (2010), who explored worth of head measurements for drawdown and flux predictions.

In this paper we demonstrate how the analysis can be extended to the context of optimizing a regional groundwater level monitoring network. The network selected for this demonstration comprises the dataset used in the Lockyer Valley model calibration (note that this network has since been extended beyond that available at the time of calibration). The model is being used to assess the potential use of Purified Recycled Water (PRW) to supplement water supplies within the Lockyer Valley in South East Queensland. The target for the monitoring network optimization is to improve the reliability of pertinent predictions made by this model. While a range of PRW demand predictions are being considered, this paper restricts discussion to the predicted PRW demand required to maintain a target groundwater level surface, where this target surface is known to sustain creek flows, and well supply reliability.

\section{DATA OPTIMISATION USING PREDICTIVE UNCERTAINTY ANALYSIS}

Data worth can be assessed in terms of the calculated reduction in model predictive uncertainty that would be accrued through inclusion of extra information furnished by a measurement. Measurements may be system state measurements such as water levels or stream flows, hydraulic property tests, or could relate to model inputs that are typically ignored in uncertainty assessments, such as recharge boundary conditions and abstraction estimates. The requirements for optimization of a monitoring data network, using the analysis presented herein are: (i) a prediction of a groundwater response and a physically based model used to make that prediction, where the model contains sufficient 'real-world' hydraulic process detail for which the prediction is sensitive; (ii) if calibration constraints are imposed, a description of the probability distribution of the error of measurements used in the calibration dataset is required and must include model structural error as evidenced by model-to-measurement misfit; (iii) a description of the probability distribution of both fixed model inputs and estimated parameters prior to imposition of calibration constraints (e.g. "a priori" distributions); and (iv) calculated sensitivities of the prediction to the measurements, model inputs and model parameters. The uncertainty analysis theory, using the data listed above, and underpinning the analysis of data worth is now introduced.

Bayesian analysis describes how measurements of system state are used to constrain the values of parameters that can be potentially used by a model, such that only those parameters which allow the model to replicate historical measurements are allowed. As outlined in Dausman et al. (2010), if noise associated with these measurements and parameters display Gaussian variability, the formulation of equations that describe the constraining effect of these measurements is relatively straightforward, as shown below.

Let $\mathbf{x}$ be a vector of multi-Gaussian random variables with a covariance matrix $\mathbf{C}(\mathbf{x})$, where $\mathbf{x}$ can be partitioned into two subvectors $\mathbf{x}_{1}$ and $\mathbf{x}_{2}$, and $\mathbf{C}(\mathbf{x})$ be partitioned on the same $\mathbf{x}_{1}, \mathbf{x}_{2}$ basis e.g. $\left(\mathrm{C}\left(\mathbf{x}_{1} \mathbf{x}_{1}\right)\right.$ or 
$\mathbf{C}_{11}, \mathrm{C}\left(\mathbf{x}_{1} \mathbf{x}_{2}\right)$ or $\mathbf{C}_{12}, \mathrm{C}\left(\mathbf{x}_{2} \mathbf{x}_{1}\right)$ or $\mathbf{C}_{21}$, and $\mathrm{C}\left(\mathbf{x}_{2} \mathbf{x}_{2}\right)$ or $\left.\mathbf{C}_{22}\right)$. If elements of $\mathbf{x}_{2}$ become known, then $\mathbf{C}_{11}^{\prime}$, the covariance matrix of $\mathbf{x}_{1}$ conditional on knowing $\mathbf{x}_{2}$ is calculated as (from Koch 1987):

$$
\mathbf{C}_{11}^{\prime}=\mathbf{C}_{11}-\mathbf{C}_{21} \mathbf{C}^{-1}{ }_{22} \mathbf{C}_{21}
$$

This formula can be used to characterize the reduction in uncertainty of model parameters (and the predictions which depend on them) that is achieved by adding data that contains information pertinent to model parameters, as is now shown.

Suppose that the $(m \times 1)$ vector $\mathbf{p}$ contains the values of parameters used by a model. Let the action of a (linear) model on these parameters $\mathbf{p}$ be represented by the matrix $\mathbf{X}$. Unless the parameter values encapsulated in the vector $\mathbf{p}$ are accurately known at all places within a model domain, they must be described in probabilistic terms. Therefore, let the covariance matrix of $\mathbf{p}$ be denoted as $\mathbf{C}(\mathbf{p})$. Further, let the vector $\mathbf{h}$ represent observations of system state comprising the model calibration set and allow for these observations to be contaminated by measurement noise $\boldsymbol{\varepsilon}$, such that:

$$
\mathbf{h}=\mathbf{X p}+\boldsymbol{\varepsilon}
$$

Let $s$ (a scalar) designate a prediction made by the model; let the sensitivities of this prediction to model parameters be represented by the vector $\mathbf{y}$. Then $s$ is calculable using the relationship:-

$$
s=\mathbf{y}^{\mathbf{t}} \mathbf{p}
$$

The estimated parameter vector $\mathbf{p}$ can be determined as follows:-

$$
\mathbf{p}=\left(\mathbf{X}^{\mathrm{t}} \mathbf{Q X}\right)^{-1} \mathbf{X}^{\mathrm{t}} \mathbf{Q h}
$$

where $\mathbf{p}$ is the vector of estimated parameters and observations weights are encapsulated in the matrix $\mathbf{Q}$. The potential wrongness of estimated parameters $\mathbf{p}$ compared to those representing the true parameter field $\mathbf{p}$, can be described as: -

$$
\mathbf{p}-\mathbf{p}=\mathbf{I p}-\left(\mathbf{X}^{\mathrm{t}} \mathbf{Q X}\right)^{-1} \mathbf{X}^{\mathrm{t}} \mathbf{Q h}
$$

Then the covariance of parameter error, $C(\mathbf{p}-\mathbf{p})$ is easily calculated from (4) (as in Menke, 1984) as:-

$$
\mathrm{C}(\mathbf{p}-\mathbf{p})=(\mathbf{I}-\mathbf{R}) \mathrm{C}(\mathbf{p})(\mathbf{I}-\mathbf{R})^{\mathrm{t}}+\mathbf{G C}(\boldsymbol{\varepsilon}) \mathbf{G}^{\mathrm{t}}
$$

where $\mathrm{R}$ is $\left(\mathbf{X}^{\mathrm{t}} \mathbf{Q X}\right)^{-1} \mathbf{X}^{\mathrm{t}} \mathbf{Q X}$ and $\mathrm{G}$ is $\left(\mathbf{X}^{\mathrm{t}} \mathbf{Q} \mathbf{X}\right)^{-1} \mathbf{X}^{\mathrm{t}} \mathbf{Q}$

And substituting for (3) in (6) we can calculate the covariance of predictive error as: -

$$
\sigma_{s-\underline{s}}^{2}=\mathbf{y}^{t}(\mathbf{I}-\mathbf{R}) \mathrm{C}(\mathbf{p})(\mathbf{I}-\mathbf{R})^{\mathrm{t}} \mathbf{y}+\mathbf{y}^{\mathrm{t}} \mathbf{G C}(\boldsymbol{\varepsilon}) \mathbf{G}^{\mathrm{t}} \mathbf{y}
$$

The formulation of this equation is described in detail in Moore and Doherty (2005), and in Doherty (2011b). In Christensen and Doherty (2008) a Bayesian formulation of equation (7) is presented, which calculates predictive uncertainty rather than error variance. The Bayesian approach abandons the notion of a calibrated model, and instead a suite of model outputs are used to describe the possible predictions, and is encapsulated as: -

$$
\sigma_{\underline{s}}^{2}=\mathbf{y}^{\mathrm{t}} \mathrm{C}(\mathbf{p}) \mathbf{y}-\mathbf{y}^{\mathrm{t}} \mathrm{C}(\mathbf{p}) \mathbf{X}^{\mathrm{t}}\left[\mathbf{X C}(\mathbf{p}) \mathbf{X}^{\mathrm{t}}+\mathbf{C}(\boldsymbol{\varepsilon})\right]^{-1} \mathbf{X C}(\mathbf{p}) \mathbf{y}
$$

This formulation has been implemented via the PEST utility software PREDUNC (Doherty 2011) and used, in this form, in this work. An important characteristic of equations (7) and (8) is that they do not contain parameter values, or measurements or model output values. Instead only the sensitivities of the model outputs to parameters under calibration and predictive conditions are considered, as constrained by the matrix $\mathbf{X}$ and the vector $\mathbf{y}$ respectively. The existing calibration dataset can therefore be augmented by proposed new data, by simply adding rows to the $\mathbf{X}$ matrix. The reduction in $\sigma_{\mathrm{s}}^{2}$ resulting from such additions to the $\mathbf{X}$ matrix is a measure of the worth of such additions to the calibration dataset; this is the metric for observation worth that is employed in this paper.

Similarly, to assess the contribution of a parameter to the uncertainty of a model output, $\mathbf{C}(\mathbf{p})$ can be replaced by $\mathbf{C}^{*}(\mathbf{p})$, where the parameters can be assumed to have a zero variance if measured, or a reduced variance as 
calculated using equation (6) or its Bayesian equivalent encapsulated in equation (8). In this case, the reduction in the uncertainty associated with a prediction $s$ as a result of improved knowledge of these parameters is calculated as:

$$
\Delta \sigma^{2}{ }_{\mathrm{s}}=\mathbf{y}^{\mathrm{t}}\left(\mathrm{C}(\mathbf{p})-\mathrm{C}^{*}(\mathbf{p})^{\mathrm{t}} \mathbf{y}\right.
$$

To apply this analysis to monitoring data network optimizations, a pertinent prediction $s$ becomes the target for which data worth is optimized. Equations (7), (8) and/or (9) are then evaluated on the basis of the addition of measurement data to be optimized and/or enhanced parameter knowledge respectively.

\section{CASE STUDY: LOCKYER VALLEY, SOUTH EAST QUEENSLAND}

The Lockyer Valley is located approximately $80 \mathrm{~km}$ west of Brisbane and is a heavily used agricultural catchment which contributes significantly to the vegetable supply for Brisbane. Recharge to this alluvial groundwater system predominantly occurs from leakage from the surface water ways within the Lockyer catchment; however diffuse recharge from rainfall and irrigation drainage also contributes to the water balance of this alluvial groundwater system (RPS 2010). Long term drawdowns in groundwater levels, and the resulting impacts of reduced surface water flows, and well yields, has motivated an investigation of potential supplementary water supplies to the Valley.
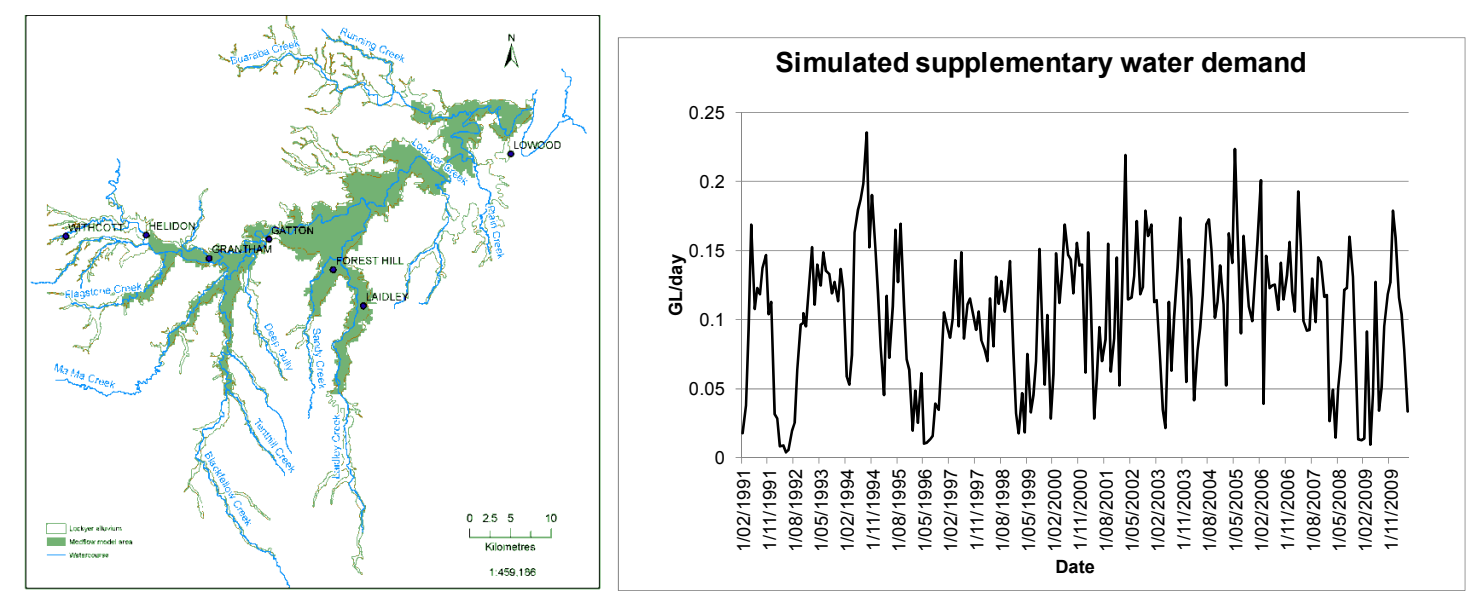

Figure1. a) Extent of the Lockyer Valley numerical groundwater model, and b) Simulated water demand over time for a selected target groundwater level surface.

The South East Queensland (SEQ) Water Grid, which operates the third largest recycled water scheme in the world represents one potential supplementary water supply (ca. 15-37 GL/a being available for the Lockyer system). This supplementary water supply option is currently being explored in detail by government agencies and the water grid managers.

A transient, single layer, regional groundwater flow model (Arunakumaren 2003) has been extended for this exploration and has been calibrated to groundwater level data from 1991 to 2010 (refer to RPS 2010 for details of this model). The model domain area is depicted in Figure 1a and comprises 250 columns and 228 rows with a resolution of $250 \mathrm{~m}$. The total water demand that would be required to meet a target 'high' groundwater level surface within the Valley is assessed over a 20 year period as depicted in Figure 1b; this is the predictive simulation selected to demonstrate the data worth optimization methodology in this paper,. Alternative predictive simulations are also being explored, and the resulting data worth optimizations compared with this analysis presented herein, however for simplicity they are not presented within this paper.

Table 1 lists the parameter groups considered in the calibration of the model. Note that the parameter groups listed in table 1 include both hydraulic property parameters and also model parameters that are usually 'hardwired' in the construction of the model, e.g. groundwater abstraction rates. The spatial correlation structure of the aquifer hydraulic conductivity and storage parameters was examined geostatistically, with a resulting spatial correlation scale smaller than the grid spacing scale, therefore off-diagonal terms of the $\mathbf{C}(\mathbf{p})$ matrix for these properties were assumed to be zero. For the remaining 'hardwired' parameters, the impact of general errors in their estimation was assessed in terms of a range of array multipliers. The $95 \%$ confidence range of these multipliers was estimated to be from 0.5 and 1.5. Using the model, calculated 
C. Moore et al. Optimisation of monitoring data for increased predictive reliability ...

sensitivities, the existing calibration dataset as weighted by model-to-measurement misfits, and the 'a priori' parameter distributions listed in Table 1, data worth was examined in terms of the selected prediction.

Table 1. 'A priori' model parameter ranges

\begin{tabular}{ccc}
\hline Parameter Group & $\mathbf{9 5 \%}$ confidence limit range & $\begin{array}{c}\text { Parameter standard } \\
\text { deviation }\end{array}$ \\
\hline Recharge (Creek and Diffuse) & $\begin{array}{c}0.5 \text { to } 1.5 \text { (multiplier of the } \\
\text { current parameter distributions) }\end{array}$ & $\begin{array}{c}0.25 \text { (multiplier of the current } \\
\text { parameter distributions) } \\
\text { Abstraction }\end{array}$ \\
$\begin{array}{c}0.5 \text { to } 1.5 \text { (multiplier of the } \\
\text { current parameter distributions) }\end{array}$ & $\begin{array}{c}0.25 \text { (multiplier of the current } \\
\text { parameter distributions) }\end{array}$ \\
Hydraulic conductivity & $1-10000 \mathrm{~m} /$ day & 0.9 (log domain) \\
Storage & 0.003 to 0.3 & 1 (log domain) \\
\hline
\end{tabular}

\section{RESULTS AND DISCUSSION}

The analyses outlined in Section 2 was firstly used to assess the relative worth of data within the groundwater level monitoring network used for the model calibration. This analysis was assessed in two ways, firstly in terms of the increase of uncertainty that occurs if an observation is removed (i.e. starting conditions assume all observations are available), and secondly in terms of the reduction of uncertainty that occurs by adding an observation to the dataset (i.e. starting conditions assume no observations are available). In both of these cases the observations can be existing or nominal future observations, as the actual value of the observation is not used in the uncertainty calculation. In this example we used the existing groundwater level monitoring network, which is reasonably extensive.

Figure 3a) describes data worth calculated on the basis that all observations have been used to constrain a prediction, and then each observation well's data has been systematically removed. The greatest uncertainty reduction is around 0.3 of the original uncertainty, and is shown by pale shading, and the least uncertainty reduction (e.g. around 1) is indicated by dark shading. This figure indicates that each single water level has greatest value in the areas where groundwater abstraction is most densely occurring (abstraction bores are shown as yellow points in Figure 3). In situations of data scarcity the data worth analysis derived from adding or removing an observation can be similar. However in this case, Figure $3 \mathrm{~b}$ indicates that each observation removed has very little effect on the prediction uncertainty, with the exception of the one part of the Lockyer where monitoring bores are scarce (monitoring bores are shown as pale grey points in Figure 3). Therefore Figure $3 \mathrm{~b}$ indicates, that there is more than sufficient water level data for the assessment of the total required water imports for a given target groundwater level, except in the Tenthill Creek area.

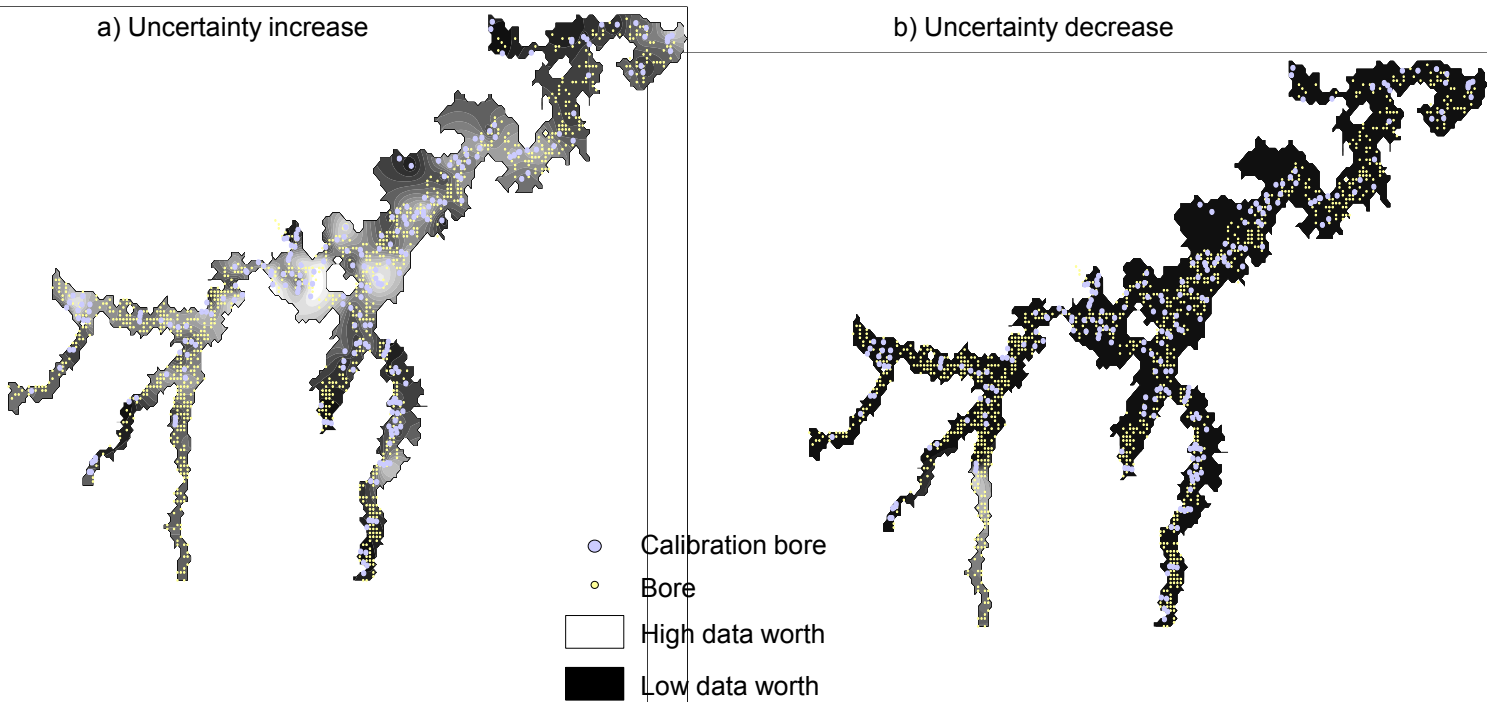

Figure 2. Plot of spatial distribution of data worth of monitored groundwater level records :a) data worth determined by beginning with no observations and adding an observation one at a time; $b$ ) data worth determined by beginning with a set of observations and removing an observation one at a time. 
It is important to note that differing environmental targets are being considered in the wider project beyond that presented in this paper. For differing targets, the existing water level monitoring data maybe be less than sufficient, for example in terms of predicting areas of ponding that could occur with large volumes of supplementary water supplies introduced to the Valley. The relative worth of a range of different data types is currently being investigated in the same manner described above, including isotope measurements, pumping tests for direct hydraulic property measurements and flood recession groundwater level measurements. The investigation of data network optimization in terms of these alternative predictions and data types is in progress.

Secondly, the extent to which acquisition of better knowledge of different types of parameters and model inputs would reduce the uncertainty of the prediction of current interest was explored, as is depicted in Figure 3. This analysis can be applied to the model with and without imposition of calibration constraints, and therefore pertain to both a pre- and post-calibrated model. (Recall that the analysis requires only sensitivities of model outputs with respect to parameters and not the values of these model outputs or of the parameters themselves).

There is some reduction in prediction uncertainty achieved from the imposition of calibration constraints depicted in Figure 3. Interestingly the analysis indicates that the calibration process has increased the extent to which predictive uncertainty can be lowered through better knowledge of hydraulic conductivity. This occurs because calibration constraints have enforced a linkage between hydraulic conductivities and other parameters. On the other hand, the prediction is principally sensitive to parameters other than hydraulic conductivity, for example specific yield, groundwater abstraction and recharge. The post calibration linkage of these parameters therefore allows knowledge of hydraulic conductivity to reduce the uncertainty of the prediction as a result of its ability to reduce the uncertainty of parameters on which the prediction more directly depends.

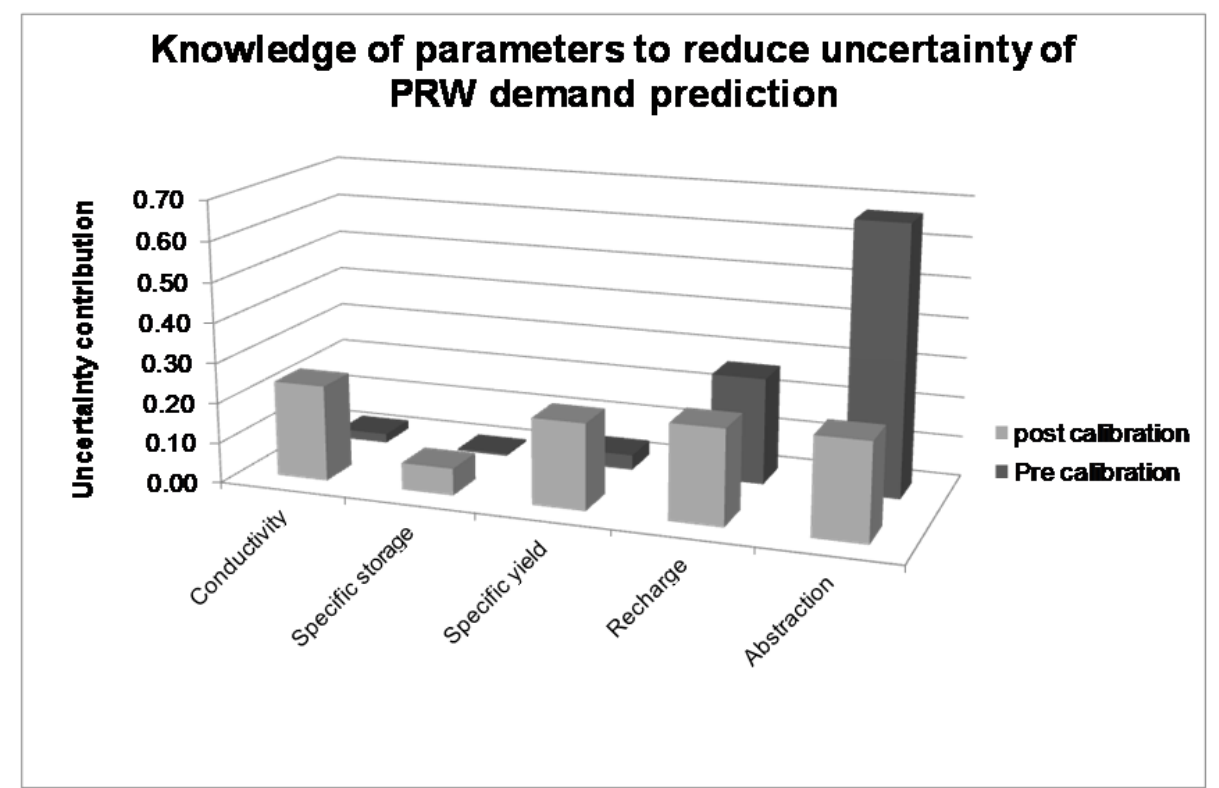

Figure 3. Summary of reduction in prediction uncertainty possible with enhanced knowledge of model parameters

\section{CONCLUSIONS AND RECOMMENDATIONS}

The optimization of a monitoring data network is able to be achieved on the basis of estimating the ability of measured data to improve predictive reliability. The efficiencies of this methodology allow it to be implemented for even large scale groundwater modeling problems. This study has demonstrated that the proposed methods have a significant potential to evaluate, extend, and optimize monitoring networks under the 'best return for investment' principle. Best return, in this case, is defined in terms of greatest improvement in predictive reliability. The method demonstrated is model independent and applicable to a wide range of observation networks. 
C. Moore et al. Optimisation of monitoring data for increased predictive reliability ...

The paper has demonstrated the utility of a methodology in the context of a specific model prediction within the Lockyer Valley. Supplementary water demand was explored in terms of the amount of water required to maintain a 'high' groundwater level surface target, over variable climate conditions. Examination and optimization of data worth for the investigation of the Lockyer PRW demand is ongoing. The investigation of data worth for more detailed predictions, and for differing data types will likely yield new information beyond that discussed in this paper.

\section{ACKNOWLEDGEMENTS}

The work of Jerome Arunamukaren \& Peter Evans of RPS Consultants and the cooperation with Ashley Bleakley of (DERM) is gratefully acknowledged.

\section{REFERENCES}

Arunakumaren, N.J and Evans, P.A, 2003 Agricultural Use of Recycled Wastewater: Hydrogeological Modelling,Water, December, pp 30 - 36

Christensen, S. and Doherty J.D. (2008), Predictive error dependencies when using pilot points and singular value decomposition in groundwater model calibration. Advances in Water Resources, 31, 674-700.

Dausman, A.M., Doherty, J., Langevin, C.D., Sukop, M.C. (2010), Quantifying Data Worth Toward Reducing Predictive Uncertainty. Ground Water, Vol, 48, No. 5, pp 729-740.

Doherty, J. (2011a). PEST: Model-Independent Parameter Estimation. Brisbane, Australia.: Watermark Numerical Computing.

Doherty, J. (2011b). PEST: Groundwater Data Utilities. Brisbane, Australia.: Watermark Numerical Computing.

Fienen, M. N., Doherty, J. E., Hunt, R. J., and Reeves, H. W., (2010), Using Prediction Uncertainty Analysis to Design Hydrologic Monitoring Networks: Example Applications from the Great Lakes Water Availability Pilot Project: U. S. Geological Survey Scientific Investigations Report 2010-5159, 44 p. [http://pubs.usgs.gov/sir/2010/5159]

Koch, K. R. (1987), Parameter Estimation and Hypothesis Testing in Linear Models. Springer-Verlag, Berlin. Menke, W. (1984). Geophysical Data Analysis: Discrete Inverse Theory. International Geophysics Series no. 45, Academic Press Inc.

Moore, C. (2005). The use of regularized inversion in groundwater model calibration and prediction uncertainty analysis. Thesis submitted for the degree of Doctor of Philosophy at The University of Queensland, Australia.

Moore, C., and Doherty, D. (2005). Role of the calibration process in reducing model predictive error. Water Resources Research 41, no.5 W05050.

RPS Consult (2010): Lockyer Valley Groundwater Modelling Sensitivity Analysis, 61p, Report to the UWSRA Alliance, Brisbane, Australia.

Turnadge, C. (2010). A Predictive Uncertainty-Based Analysis of Data Worth for a Simple Groundwater Model. Thesis submitted for the degree of Bachelor of Science (Honours) in Groundwater Hydrology, Flinders University, Australia. 